\title{
Robust Optimization of H-arm Suspension Compliance Characteristics Based on Isight and CarSim
}

\author{
Shuhua Liao, a, Kaibin Cao ${ }^{1, b}$, Jinwu Liu ${ }^{2}$, Jiong Li ${ }^{1}$, Shian Yang ${ }^{2}$ \\ ${ }^{1}$ College of Automobile and Transportation, Guangxi University of Science and Technology, China; \\ ${ }^{2}$ Liuzhou KH Automotive Technologies Co. Ltd, Liuzhou 545006, China. \\ aliaohsh60@163.com, ’501333069@qq.com
}

Keywords: Robustness, Compliance Characteristics, NSGA-II, Isight, CarSim

\begin{abstract}
Pointing to problem that the comprehensive evaluation score is not ideal for the steady state cornering test conditions and the fish hook test conditions of a passenger vehicle, considering the error of speed and locations of center of mass, taking the $\mathrm{H}$-arm suspension of the passenger vehicle as an example, the robust optimization of suspension compliance characteristics based on Isight and CarSim method is proposed. Using CarSim software to model the whole vehicle and by handling stability to test the accuracy of the whole vehicle. Utilizing Isight software to analyze the degree of influence of bushing stiffness on suspension compliance characteristics and to verify the target function. Adopting the NSGA-II algorithm to optimize the multi-objective robustness of vehicle handling stability. The result illustrated that both the vehicle handling stability and robustness improved a lot after optimization.
\end{abstract}

\section{Introduction}

Suspension Compliance Characteristics means when tires get the lateral force, longitudinal force and aligning torque, the wheel alignment parameters arise from the elastic deformation of bushings would change with these force and torque [1]. Most of the suspension of vehicles uses bushings now. Suspension compliance characteristics have important influences on the vehicle handling stability [2]. The influence of hard point of Macpherson on vehicle handling stability is studied in literature [3]. Adopt the Taguchi method to optimize K\&C characteristics in literatures [4]. In this paper, the vehicle handling stability of a passenger vehicle with $\mathrm{H}$-arm suspension is the research object, aiming at the problems of unapparent performance improvement of sample vehicles in the chassis tuning process, the whole vehicle model is established by using Carsim, and the sensitivity analysis of the $\mathrm{H}$-arm suspension compliance characteristics are carried out with Isight and Matlab. The approximate model between the system variable and the target response is obtained by the RSM method. Adopt the NSGA-II algorithm to optimize the multi-objective robustness of vehicle handling stability to explore an effective method to improve vehicle handling stability.

\section{Establishment and verification of vehicle model}

\subsection{Establishment of vehicle model}

In this paper, the simulation model of a vehicle is established in CarSim by measuring the $\mathrm{K} \& \mathrm{C}$ characteristic test data. CarSim software is a multi-body dynamics software based on system characteristic parameter modeling, and mainly consists of three parts, model input, analysis process and result output. In the modeling, input the basic parameters of the whole vehicle, steering system, braking system, $\mathrm{K} \& \mathrm{C}$ characteristics of the front and rear suspension and the relevant parameter information of tire. The system defaults are used in aerodynamics, engine parameters, braking systems and driveline systems. Part parameters of the vehicle model as shown in Tab.1. 
Tab.1 Part parameters of the whole vehicle model

\begin{tabular}{|c|c|c|c|}
\hline Parameter & Value & Parameter & Value \\
\hline Height $/ \mathrm{mm}$ & 1750 & Sprung Mass/kg & 1580 \\
\hline Width/mm & 1820 & Front Wheel Load $/ \mathrm{kg}$ & 955 \\
\hline Wheel Base/mm & 2750 & Rear Wheel Load $/ \mathrm{kg}$ & 853 \\
\hline Front Wheel Track/mm & 1560 & Front Suspension Stiffness/ N*mm ${ }^{-1}$ & 32 \\
\hline Rear Wheel Track /mm & 1556 & Rear Suspension Stiffness / N*mm & 84 \\
\hline Height of Mass Center /mm & 630 & Effective Wheel Rolling Radius/mm & 350 \\
\hline
\end{tabular}

\subsection{Simulation and test verification of vehicle model}

According to GB/T 6323-2014 Vehicle Handling and Stability Test Method [5], the vehicle model is simulated in CarSim, including Steady-state cornering test, Steering wheel angle step test, steering angle impulse test and Fish hook test. By actual Handling Stability Test to sample cars, compare experiment results with simulation results to test the accuracy of the model. It can be seen from Fig.1-3, the steering wheel angle, yaw rate and lateral acceleration curve and the test curve contrast under the fish hook test conditions, but the trend is consistent. This is mainly due to the estimation of the inertia of the steering system, the difference between the tire model and the actual model, so it can be judged that the simulation model is accurate. To contrastively analyze the other condition of handling stability, they all have satisfactory accuracy.

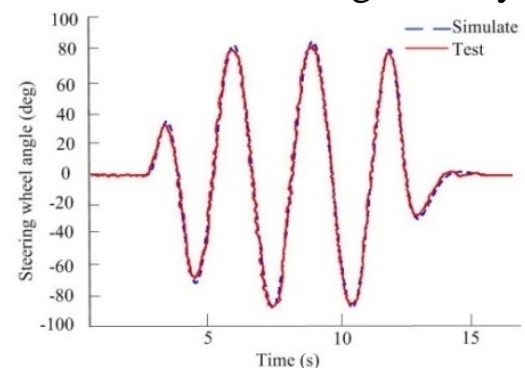

Fig.1 Steering Wheel Angle Curve

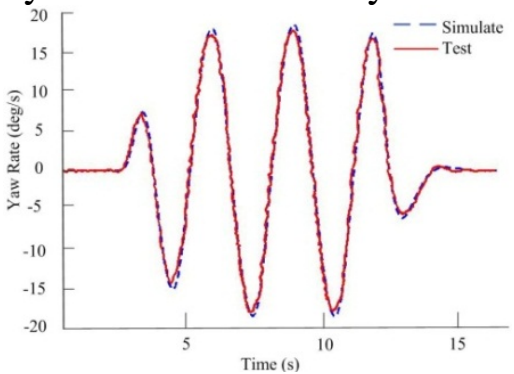

Fig.2 Yaw Rate Curve

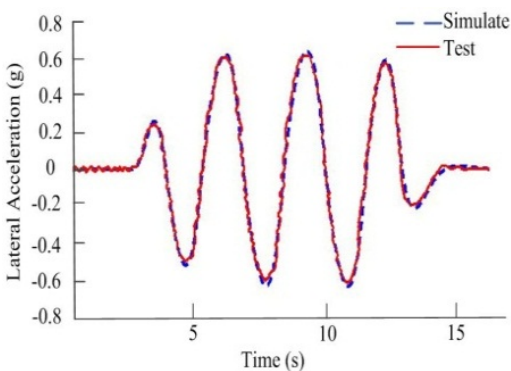

Fig.3 Lateral Acceleration Curve

\section{Optimization principles}

Isight is one of the multi-disciplinary design optimization software based on parameters. This paper integrates CarSim and Matlab with Isight platform. Define optimization parameter, initialize parameters and constraints in Isight, and the appropriate optimization algorithm is selected according to the type of optimization problem. In MATLAB, the optimization parameters are processed in advance, and then the parameters needed for the whole vehicle simulation are passed to CarSim. The CarSim solver is called to carry out the simulation test condition of the whole vehicle. Choose a suitable approximate model to fit the results of simulation test. According to the QC/T 480-1999 Vehicle Handling and Stability Index Limit and Evaluation Method [6], evaluate the parameter values of the vehicle handling stability index, such as car yaw rate, lateral acceleration and steering wheel angle. Output parameters from CarSim and passed to Matlab for post-processing, and Matlab returns Isight to optimize the iteration function and update parameters of each step in real time.

\section{Robust Optimization of suspension compliance characteristics}

\subsection{Sensitivity Analysis}

The sensitivity Analysis of $\mathrm{H}$-arm suspension compliance characteristics is through co-simulation of Isight, CarSim and Matlab software. Utilizing Optimal Latin hypercube to design experiment and find remarkable elements of on the lateral acceleration, yaw velocity and body roll angle of the vehicle under the steady-state cornering and the fish hook test conditions in Isight software. And evaluate scoring in Matlab according to the literature [6]. Taking H-arm suspension compliance characteristics as variable, analyze handling and stability of the whole vehicle under the steady-state cornering and the fish hook test conditions. The comprehensive evaluation score of the steady-state cornering test $N_{w}$ and the comprehensive evaluation score of the fish hook simulation 
test Ns are objective responses. Analyze the sensitivity of the nine parameters of the $\mathrm{H}$-arm suspension compliance characteristics. The parameter variables with sensitivity above $10 \%$ were considered as influencing factors, and the number of impacts was listed as shown in Tab.2. Set the initial value of the $\mathrm{H}$-arm suspension compliance characteristics and use its variation coefficient as the independent variable, which is 1 initially, variation range is $(0.5,2)$.

Record: $\mathrm{N}_{\mathrm{w}}=$ Comprehensive evaluation score of the steady-state cornering test

Ns= Comprehensive evaluation score of the fish hook simulation test

$\mathrm{F}_{1}=\mathrm{H}_{\text {_ }}$ aligningtorque_camberratemean

$\mathrm{F}_{2}=\mathrm{H} \_$aligningtorque_toeratemean

$\mathrm{F}_{3}=\mathrm{H}$ _ lateralforce_camberratemean

$\mathrm{F}_{4}=\mathrm{H}_{-}$lateralforce_toeratemean

$\mathrm{F}_{5}=\mathrm{H} \_$lateralforce_trackratemean

$\mathrm{F}_{7}=\mathrm{H}_{-}$longitudinalforce_camberratemean

$\mathrm{F}_{6}=\mathrm{H}_{-}$longitudinalforce_baseratemean

$\mathrm{F}_{9}=\mathrm{H} \_$longitudinalforce_toeratemean

$\mathrm{F}_{8}=\mathrm{H}_{-}$longitudinalforce_casteratemean

Tab.2 Suspension Compliance Characteristics Sensitivity Analysis Results

\begin{tabular}{|c|c|c|c|c|c|c|c|c|c|}
\hline $\mathrm{N}^{\mathrm{F}}$ & $\mathrm{F}_{1}$ & $\mathrm{~F}_{2}$ & $\mathrm{~F}_{3}$ & $\mathrm{~F}_{4}$ & $\mathrm{~F}_{5}$ & $\mathrm{~F}_{6}$ & $\mathrm{~F}_{7}$ & $\mathrm{~F}_{8}$ & $\mathrm{~F}_{9}$ \\
\hline $\mathrm{N}_{\mathrm{w}} / \%$ & 2.34 & 17.39 & -1.20 & 25.26 & -11.57 & 4.36 & -8.14 & 0.48 & 29.26 \\
\hline $\mathrm{Ns} / \%$ & 1.86 & 4.86 & 26.02 & -10.84 & 26.02 & -20.95 & 6.00 & 1.91 & -1.53 \\
\hline Numble & 0 & 1 & 1 & 2 & 2 & 1 & 0 & 0 & 1
\end{tabular}

According to Tab.2, parameter variables $F_{2}, F_{3}, F_{4}, F_{5}, F_{6}$, and $F_{9}$, which affect the number above zero, are put as controllable factors. At the same time, considering the state of the vehicle in the actual test condition, the height of mass center and the distance of mass center from the front axle are taken as noise factors, and the error changes between plus or minus $20 \mathrm{~mm}$ based on the original mass center coordinates.

\subsection{Determine the objective function}

\subsubsection{Steady-state cornering test}

According to the literature [6], the steady-state cornering features are evaluated and scored.

1) The evaluation score of the lateral acceleration value of a Neutral turning point is defined as $\mathrm{Na}_{\mathrm{n}}$. When no neutral point occurs, the score is recorded as 100 .

$$
N a_{\mathrm{n}}=60+\frac{40}{a_{\mathrm{n} 100}-a_{\mathrm{n} 60}} \cdot\left(a_{n}-a_{n 60}\right)
$$

In the formula, the $a_{\mathrm{n}}$ is the lateral acceleration value of the slope of zero at the relation curve between the difference of the slip angle between the front axle and rear axles and the lateral acceleration. The $a_{\mathrm{n} 100}$ and $a_{\mathrm{n} 60}$ are respectively the upper and lower values of the lateral acceleration of the neutral turning point.

2) The evaluation score value of Under-steer degree is defined as $\mathrm{N}_{U}$.

$$
\begin{gathered}
\lambda=60+\frac{2 \cdot U_{60} / U_{100}}{U_{60} / U_{100}-2} \cdot U_{100} \\
N_{\mathrm{U}}=60+\frac{U\left(U_{60}-U\right)(\lambda-U)}{U_{100}\left(U_{60}-U_{100}\right)\left(\lambda-U_{100}\right)} \cdot 40
\end{gathered}
$$

In the formula, $\lambda$ is the ratio coefficient, $U$ is the test value of the under-steer degree, and is the slope value of the lateral acceleration at the $2 \mathrm{~m} / \mathrm{s}^{2}$ point between the front and rear axles slip angles difference and the lateral acceleration curve. The $U_{100}$ and $U_{60}$ are respectively the upper and lower values of the Under-steer degree.

3) The evaluation score value of Carriage reclining degree is defined as $\mathrm{N}_{\phi}$.

$$
N_{\phi}=60+\frac{40}{K_{\phi 60}-K_{\phi 100}} \cdot\left(K_{\phi 60}-K_{\phi}\right)
$$

In the formula, $K_{\phi}$ is the test value of the Carriage reclining degree, and is the slope value of the lateral acceleration at the $2 \mathrm{~m} / \mathrm{s}^{2}$ point between the roll angle and the lateral acceleration curve. The $K_{\phi 100}$ and $K_{\phi 60}$ are respectively the upper and lower values of the Carriage reclining degree.

The comprehensive evaluation score of the steady-state cornering simulation test is defined as $N_{w}$ and calculated according to formula (5).

$$
N_{w}=\frac{N a_{n}+N_{U}+N_{\phi}}{3}
$$

\subsubsection{Fish hook test}

According to the literature [6], the Fish hook test characteristics are evaluated and scored.

1) The evaluation score of the average peak value of steering wheel angle is defined as $N_{\theta}$. 


$$
N_{\theta}=60+\frac{40}{\theta_{60}-\theta_{100}} \cdot\left(\theta_{60}-\theta\right)
$$

In the formula, $\theta$ is the test value of the average peak value of steering wheel angle at $65 \mathrm{~km} / \mathrm{h}$ speed. The $\theta_{100}$ and $\theta_{60}$ are respectively the upper and lower values of the average peak value of steering wheel angle.

2) The evaluation score of the average peak value of yaw rate is defined as $\mathrm{N}_{\mathrm{r}}$.

$$
N_{r}=60+\frac{40}{r_{60}-r_{100}} \cdot\left(r_{60}-r\right)
$$

In the formula, $r$ is the test value of the average peak value of yaw rate at $65 \mathrm{~km} / \mathrm{h}$ speed. The $\mathrm{r}_{100}$ and $r_{60}$ are respectively the upper and lower values of the average peak value of yaw rate.

The comprehensive evaluation score of the fish hook simulation test is defined as $N_{s}$ and calculated according to formula (8).

$$
N_{\mathrm{S}}=\frac{N_{\theta}+2 N_{r}}{3}
$$

Here the comprehensive evaluation scores of the Steady-state cornering and the Fish hook tests are $N_{w}$ and $N s$ as objective functions. The smaller objective function response, the better it is.

Considering competing vehicle database and determining the ideal ranges of the comprehensive evaluation score of the Steady-state cornering and the Fish hook test are $(90,100)$.

\subsubsection{RSM Fitting of Objective Function}

According to the results of sensitivity analysis, the $N_{w}$ and $N s$ objective functions are fitted respectively by using RSM in Isight. The approximation model obtained by regression analysis indicates the relationship between the suspension system variable and the objective response.

The fitting objective function results of the comprehensive evaluation score of the Steady-state cornering test $N_{w}$ as shown in Formula (9).

$f_{1}(x)=92.895-1.752 \mathrm{x}_{1}-0.006 \mathrm{x}_{2}-0.337 \mathrm{x}_{3}-0.284 \mathrm{x}_{4}-0.358 \mathrm{x}_{5}-0.426 \mathrm{x}_{6}+1.124 \mathrm{x}_{1} \mathrm{x}_{2}+0.256 \mathrm{x}_{1} \mathrm{x}_{3}-0.019 \mathrm{x}_{1} \mathrm{x}_{4}+0.187 \mathrm{x}_{1}$ $\mathrm{x}_{5}+0.094 \mathrm{x}_{1} \mathrm{x}_{6}+0.153 \mathrm{x}_{2} \mathrm{x}_{3}+0.179 \mathrm{x}_{2} \mathrm{x}_{4}-0.071 \mathrm{x}_{2} \mathrm{x}_{5}-0.013 \mathrm{x}_{2} \mathrm{x}_{6}+0.307 \mathrm{x}_{3} \mathrm{x}_{4}-0.824 \mathrm{x}_{3} \mathrm{x}_{5}+0.751 \mathrm{x}_{3} \mathrm{x}_{6}+1.425 \mathrm{x}_{4} \mathrm{x}_{5}-0$. $\begin{array}{llllllllll}945 \mathrm{x}_{4} \mathrm{x}_{6}+1.729 \mathrm{x}_{5} \mathrm{x}_{6}+2.325 \mathrm{x}_{1}^{2} & -0.581 \mathrm{x}_{2} & 2 & -0.774 \mathrm{x}_{2} & 2 & -0.589 \mathrm{x}_{3}^{2} & -0.014 \mathrm{x}_{4} & 2 & -0.031 \mathrm{x}_{6}^{2} & 2\end{array}$

The fitting objective function results of the comprehensive evaluation score of the Fish hook test Ns as shown in Formula (10).

$f_{2}(x)=92.895-1.874 \mathrm{x}_{1}-0.002 \mathrm{x}_{2}-0.348 \mathrm{x}_{3}-0.301 \mathrm{x}_{4}-0.362 \mathrm{x}_{5}-0561 \mathrm{x}_{6}+1.215 \mathrm{x}_{1} \mathrm{x}_{2}-0.466 \mathrm{x}_{1} \mathrm{x}_{3}+0.087 \mathrm{x}_{1} \mathrm{x}_{4}+0.207 \mathrm{x}$ ${ }_{1} \mathrm{x}_{5}-0.105 \mathrm{x}_{1} \mathrm{x}_{6}+0.243 \mathrm{x}_{2} \mathrm{x}_{3}-0.228 \mathrm{x}_{2} \mathrm{x}_{4}+0.123 \mathrm{x}_{2} \mathrm{x}_{5}-0.054 \mathrm{x}_{2} \mathrm{x}_{6}+0.546 \mathrm{x}_{3} \mathrm{x}_{4}-0.533 \mathrm{x}_{3} \mathrm{x}_{5}-0.863 \mathrm{x}_{3} \mathrm{x}_{6}+1.755 \mathrm{x}_{4} \mathrm{x}_{5}+1$.

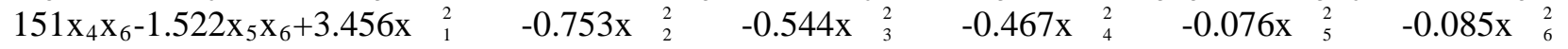
(10)

By examining the fitting effect, the fitted index values are shown in Tab.3.

Tab.3 Fit goodness index results

\begin{tabular}{|c|c|c|c|}
\hline Objective Function & $R^{2}$ & $R_{\mathrm{adj}}^{2}$ & $R_{\mathrm{e}}$ \\
\hline$f_{1}(x)$ & 0.942 & 0.921 & $8.154 \mathrm{E}-8$ \\
\hline$f_{2}(x)$ & 0.935 & 0.918 & $5.732 \mathrm{E}-7$ \\
\hline
\end{tabular}

Among them, the coefficient $R^{2}$ is closer to 1 , which shows that the higher the goodness of fit. The better the fit is, $R^{2}$ is usually greater than 0.9 . And the correction coefficient $R_{\text {adj }}^{2}$ is closer to 1 , which shows that the fitting effect is better. The Re reflects the residual of the fitting term, the smaller it is, the better the fitting effect. And the general requirement Re value is less than 0.02. It can be seen from tab.2 that the fitting effect is more ideal.

\subsection{Robust optimization based on NSGA-II algorithm}

Based on the results of RSM fitting obtained in section 4.2.3, adopt the NSGA-II algorithm to optimize the multi-objective robustness of vehicle handling stability. And the multi-objective optimization model as shown in Formula (11).

$$
\left\{\begin{array}{l}
\max f_{1}(x) \\
\max f_{2}(x) \\
90 \leq f_{1}(x) \leq 100 \\
90 \leq f_{2}(x) \leq 100
\end{array}\right.
$$

The maximum mean and minimum variance of $N_{w}$ and $N s$ are considered as optimization objectives. Set the NSGA-II parameters, the population number is 40 , genetic algebra is 50 , and the crossover probability is 0.9 . After calculation and analysis, the Pareto optimal solution set is shown 
in Fig.4.

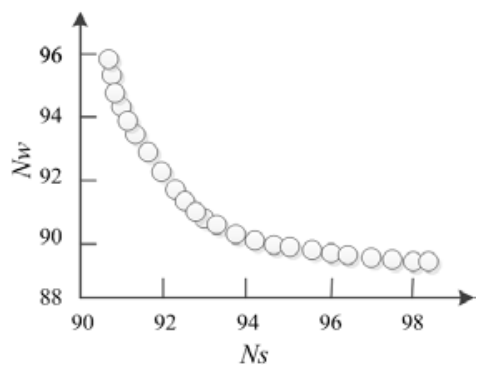

Fig.4 Pareto optimal solution set distribution

As Fig.4 shows, there is a conflict between the objective function $f_{1}(x)$ and $f_{2}(x)$. It is more suitable to select the Pareto Optimal solution of intermediate section. The final solution of this optimization is compared with the initial value of the optimization variable, as shown in Tab.4.

Tab.4 Robustness optimization before and after the design variable value

\begin{tabular}{|c|c|c|c|}
\hline Variable & Unit & Before & After \\
\hline $\mathrm{F}_{2}$ & $\mathrm{deg} /(\mathrm{N} \cdot \mathrm{mm})$ & $-1.065 \mathrm{E}-06$ & $-6.934 \mathrm{E}-07$ \\
\hline $\mathrm{F}_{3}$ & $\mathrm{deg} / \mathrm{N}$ & $-2.936 \mathrm{E}-04$ & $-2.521 \mathrm{E}-04$ \\
\hline $\mathrm{F}_{4}$ & $\mathrm{deg} / \mathrm{N}$ & $-7.206 \mathrm{E}-05$ & $-3.872 \mathrm{E}-05$ \\
\hline $\mathrm{F}_{5}$ & $\mathrm{~mm} / \mathrm{N}$ & $-1.745 \mathrm{E}-04$ & $-1.253 \mathrm{E}-04$ \\
\hline $\mathrm{F}_{6}$ & $\mathrm{~mm} / \mathrm{N}$ & $-1.837 \mathrm{E}-03$ & $-1.190 \mathrm{E}-03$ \\
\hline $\mathrm{F}_{9}$ & $\mathrm{deg} / \mathrm{N}$ & $1.404 \mathrm{E}-05$ & $-8.969 \mathrm{E}-06$ \\
\hline
\end{tabular}

\section{Analysis and verification of the results}

The result of robust optimization compares with the initial value of the comprehensive evaluation scores of the steady-state cornering and the fish hook tests are $N_{w}$ and Ns. From Tab.5, the $N_{w}$ and $N s$ are within the target range, which means there is a big improvement on the characteristics of the whole vehicle handling stability.

Tab.5 The result of optimization

\begin{tabular}{|c|c|c|c|}
\hline Optimal Object & Before & After & Target field \\
\hline$N_{w}$ & 83.63 & 91.54 & {$[90,100]$} \\
\hline$N_{s}$ & 88.72 & 93.25 & {$[90,100]$} \\
\hline
\end{tabular}

Utilized simple random sampling method of the Monte Carlo for the before and after optimization to prove the validity of optimization. 1000 sample points are selected, Fig.5. Considering the error of speed and locations of center of mass, the each response variance continues to be smaller, which means the robustness of suspension of after optimization improved a lot and the effectiveness is optimized.
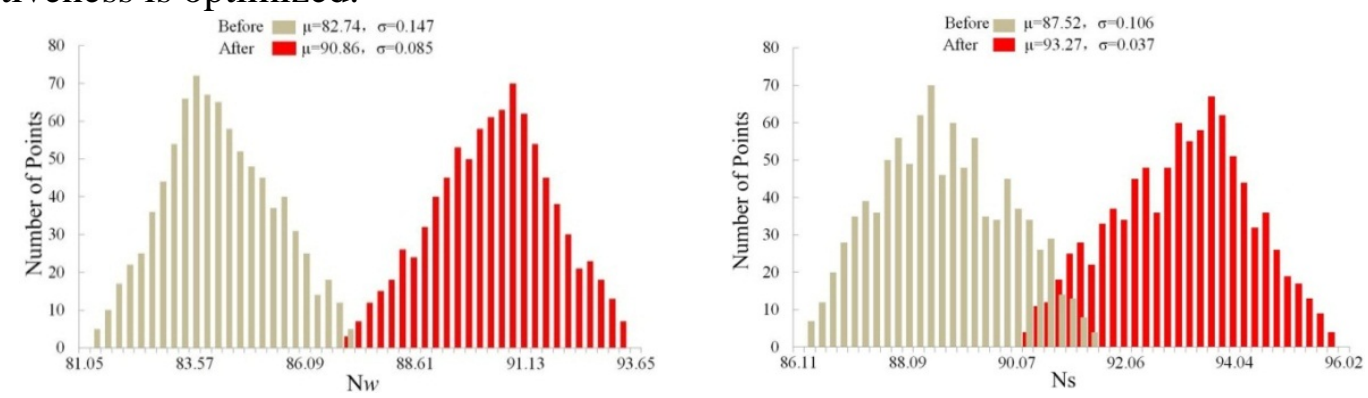

Fig.5 The probability distribution of the target before and after optimization

\section{Conclusions}

Utilized RSM to fitting the objective functions, and the second order response surface approximation model obtained by regression analysis indicates the relationship between the suspension system variable and the objective response. Adopted the NSGA-II algorithm to optimize the multi-objective robustness of vehicle handling stability. The optimization reached target range, which means the effectiveness of the method. 
By co-simulation method of Isight, CarSim and Matlab to optimize the whole vehicle handling stability in the chassis tuning process. The result illustrated that the method compared with previous method, it shortened the development cycle, raised working efficiency, and the whole vehicle handling stability are improved.

\section{References}

[1] Kang J S, Yun J R, Lee J M. Elasto-kinematic Analysis and Optimization of Suspension Compliance Characteristics. SAE Paper 970104.

[2] Gao Jin, Yang Xiujian. Sensitivity Study of Bushing Stiffness Affecting Suspension Compliance Characteristics and Transient Handling Stability[J]. Automobile Technology.2013.(11).

[3] Hongxia Jiang. Study of Front Suspension Hard point For Influence on the Vehicle Handling Stability [J]. Equipment Technology.2016.(1):45 51.

[4] Wen Fang. The Analysis and Optimization of Suspension K\&C Characters of a Car [D].Jinzhou: Liaoning University of Technology,2014.

[5] GB/T 6323-2014. Vehicle Handling and Stability Test Method.[S].

[6] QC-T 480-1999. Vehicle Handling and Stability Index Limit and Evaluation Method.[S]. 